\title{
Status of High Heat Flux Components at W7-X
}

\author{
A. Peacock ${ }^{1}$, J. Boscary ${ }^{1}$, M. Czerwinski ${ }^{2}$, G. Ehrke ${ }^{2}$, H. Greuner ${ }^{1}$, P. Junghanns ${ }^{1}$, B. Mendelevitch ${ }^{1}$, M. \\ Smirnow ${ }^{1}$, R. Stadler ${ }^{1}$, H. Tittes ${ }^{1}$, J. Tretter ${ }^{1}$ \\ ${ }^{1}$ Max Planck Institute for Plasma Physics, EURATOM Association, 85748 Garching, Germany \\ ${ }^{2}$ Max Planck Institute for Plasma Physics, EURATOM Association, 17491 Greifswald, Germany \\ Corresponding author e-mail: alan.peacock@ipp.mpg.de
}

\begin{abstract}
The actively water-cooled In-Vessel Components (IVCs) of the stellarator Wendelstein 7-X consist of the divertor, the first wall protection components, the port liners, each designed for different loading conditions, and the associated pipework, the control coils, the cryo-pump system, the Glow discharge electrodes, and a set of diagnostics. The divertor, designed for high heat fluxes, is a set of $\mathbf{1 0}$ target and baffle units arranged along the plasma surface. The design and production of these high heat flux (HHF) components is a challenging task. The divertor target elements, which are based on flat CFC (carbon-carbon fibre composite) tiles bonded via active metal casting onto $\mathrm{CuCrZr}$ cooling structures required intensive development and testing to reach a reliable performance; removing, under stationary conditions, $10 \mathrm{MW} / \mathrm{m}^{2}$.
\end{abstract}

Industrially manufactured high quality target elements have been delivered and assessed, and the process of incorporating them into assembly units, so-called modules, has begun. The time scale for the completion of the HHF divertor has been held for the last four years and the final delivery of the HHF divertor is still planned in 2017. In parallel to the realization of the divertor, most of the remaining IVCs have been defined, developed, designed and fabricated and the installation of many of these components has begun. Some of these components can also be expected, for a short period of time, to receive high heat loads approaching those of the divertor. These components will be described, in detail, from conception to realization.

Keywords-Divertor; In-Vessel Components; Baffle; First Wall; Water-cooled; W7-X; Stellarator.

\section{INTRODUCTION}

The Wendelstein 7-X (W7-X) stellarator is designed for steady state operation; the plasma being heated continuously with up to $10 \mathrm{MW}$ of ECRH (Electron-Cyclotron Resonance Heating). The plasma can also be heated with NBI (Neutral Beam Injection) and ICRH (Ion-Cyclotron Resonance Heating). The maximum available input power is $24 \mathrm{MW}$ for $10 \mathrm{~s}$. The IVCs of W7-X consist of two main components; the divertor and the first wall components. The divertor is designed to control the plasma power and particle exhaust and is subject to heat loads by convection or thermal radiation from the plasma. The first wall components protect the plasma vessel mainly from thermal radiation. Due to the long pulse length all of the IVCs are water cooled.

The different heating systems are not $100 \%$ efficient at directly heating the plasma; in an experimental device such as W7-X the plasma parameters are not always optimised to maximise the power absorbed in the plasma. Consequentially the IVCs can be directly subject to a significant fraction of the heating power. Hence, the IVCs are designed to be loaded with a wide variety of different heat fluxes, whose density is dependent upon the plasma configurations, the location of the IVCs and the different heating mechanisms (radiation or convection), as well as the heating system direct loads. The IVCs are designed in a way that they minimise the effect they have on the plasma.

ECRH stray radiation, the ECRH radiation not absorbed by the plasma, has little effect on the main IVCs as a maximum additional load of up to $5 \mathrm{~kW} / \mathrm{m}^{2}$ is expected. The exception to this statement is the cryo-pump, which although well shadowed cannot accept significant ECRH loads and the present design is under further investigation and testing.

The W7-X IVCs consist of 10 sets of HHF divertor targets, as well as 170 baffle modules, 162 heat shields, each with graphite as the plasma facing material, approximately 300 stainless steel wall protection and divertor closure panels, 136 standard and 3 graphite clad port liners, 10 control coils, 10 glow discharge electrodes and 10 cryopumps as well as the associated water supply pipework and allows for the integration of an extensive set of plasma diagnostics [1].

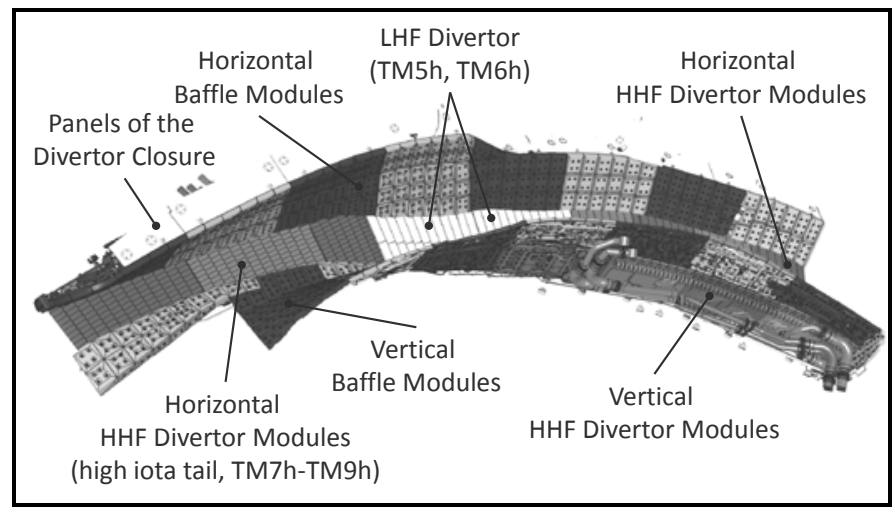

Fig. 1. Divertor overview

A broad description of the main plasma facing IVCs of the W7-X machine is given in [2]. Here the IVCs as classified with regard to their function, their position with respect to the plasma and their steady state heat loads. This paper presents how these components respond to the wider variety of heat loads mentioned above and provides details for specific components not previously described. It shows how the different standard technologies selected for W7-X, such as used in the heat shield design, have been adapted for these 
components. The heat shield design uses graphite tiles clamped with TZM bolts to CuCrZr heat sinks which are brazed to a stainless steel water cooling meander. A Sigraflex ${ }^{\circledR}$ interlayer, either 1 or $2 \mathrm{~mm}$ thick, is placed between the tiles and the heat sinks.

The divertor components, situated very close to the plasma, have been conceived to operate with steady state heat loads of up to $10 \mathrm{MW} / \mathrm{m}^{2}$, see Fig.1. The first concept for the HHF area of the divertor of W7-X was established in 1994 [3].

The realization of the HHF divertor from this time has been a long and not always problem-free process including design optimizations to reduce the highly loaded target area. The divertor design was modified so that the intermediate section of the divertor, the Lower Heat Flux (LHF) modules TM5h and TH6h do not require the use of target elements. The expected steady state load was reduced to $1 \mathrm{MW} / \mathrm{m}^{2}$ instead of 10 $\mathrm{MW} / \mathrm{m}^{2}$ but due to its proximity to the plasma transient loads of up to $6 \mathrm{MW} / \mathrm{m}^{2}$ can be foreseen.

With the clearer definition of particular plasma scenarios and a more detailed specification of the additional heating systems such as NBI, the postulated heat loads to the IVCs have evolved. It now appears that locations other than the divertor area could receive heat loads approaching those of the divertor for short periods of time. For these areas, such as parts of the baffle, where the heat shield design has been used, local heat loads of up to $500 \mathrm{~kW} / \mathrm{m}^{2}$ had previously been expected. This phenomenon of the IVCs being designed for one particular steady state heat load and being postulated yet to be subject to loads significantly higher for shorter periods of time occurs in several places in the W7-X. Design solutions used for lower steady state heat loads and higher shorter period loads are often not complementary and compromises have to be found.

This paper will address this issue for the main highly loaded IVCs of W7-X. Examples will be given for the divertor components, LHF divertor components, baffles, heat shields of the first wall and the graphite port liners.

\section{DIVERTOR COMPONENTS}

\section{A. High Heat Flux Divertor Target Elements and Modules}

The geometry of one of the 10 similar W7-X divertor units is shown in Fig. 1. The divertor consists of four main areas, three HHF areas; the vertical target, the horizontal target and the high iota tail together with the intermediate section (TM5h and TM6h) designed for a lower heat flux. The HHF areas consist of 50 to $61.5 \mathrm{~mm}$ wide target elements hydraulically and mechanically connected to each other in modules. There are 10 HHF divertor modules per divertor unit, i.e. 100 divertor modules in total. The baffle modules are located above the target modules to close the divertor volume.

The design of the HHF divertor with its extensive target area gives a high degree of experimental flexibility and 9 "reference" vacuum plasma configurations were foreseen. For some of these reference configurations, such as the limiter configuration and the high iota configuration, physics calculations showed that at low densities, heat loads of the order of $20 \mathrm{MW} / \mathrm{m}^{2}$ [4] could fall on the divertor surfaces with the nominal 10 MW ECRH input power, see Fig. 2.

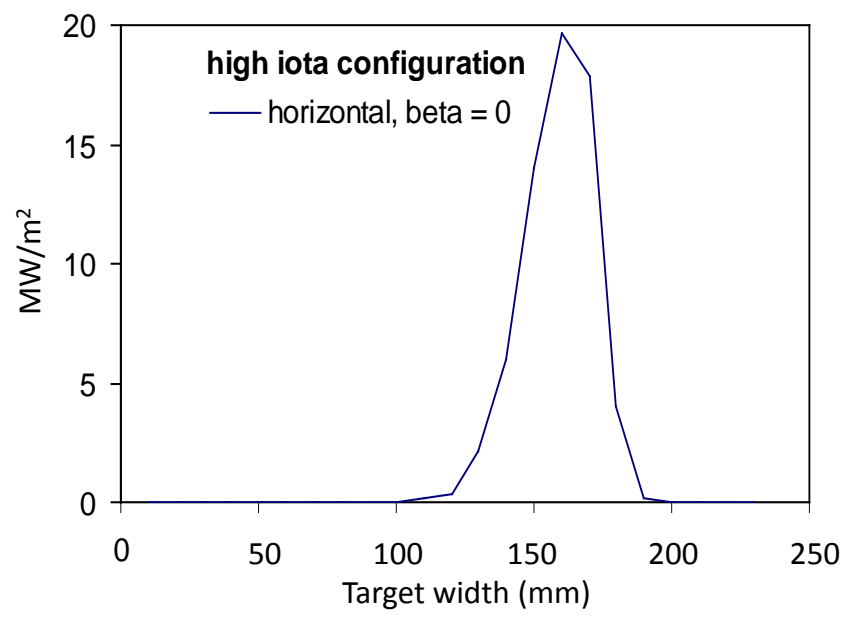

Fig. 2. Heat load footprints predicted for the horizontal (TMh7-TMh9) target area, for the high iota configuration.

For the standard configuration, one of the 9 reference configurations, $10 \mathrm{MW} / \mathrm{m}^{2}$ is predicted; the effect of plasma $\beta$ was also estimated and this tended to reduce the heat loads to the targets, see Fig.3. Methods were postulated how to reduce the heat loads to the targets, such as operating with higher densities, reducing the input powers or sweeping the strike point across the target [5]. Nevertheless it was clear that the heat loads the target elements needed to withstand be as high as possible to provide the desired experimental flexibility.

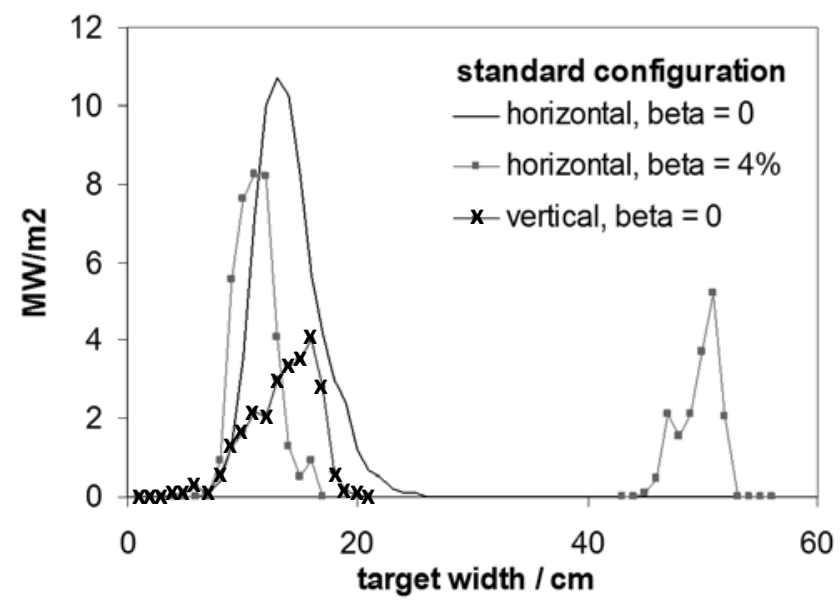

Fig. 3. Heat load footprints predicted for the main horizontal and vertical target areas, for different plasma beta values for the standard configuration.

The most developed technology with industrial experience at that time was reportedly [6] capable of removing 10 $\mathrm{MW} / \mathrm{m}^{2}$. This matched the design load requirements for the standard configuration and this technology was selected for the target elements. HHF tests of the initially developed HHF components found that the $10 \mathrm{MW} / \mathrm{m}^{2}$ limit was not, under W7-X geometry and production conditions, reliably achievable. Since the elements basically define the 
performance limits of the divertor, significant time and effort was spent in achieving this performance limit in a reliable way.

An extensive programme of testing at the HHF test facility GLADIS [7] confirmed that the finally developed $\mathrm{CFC} / \mathrm{Cu}$ joining technology used for the W7-X elements can withstand $10 \mathrm{MW} / \mathrm{m}^{2}$ [8] and tests have also shown that this quality has been transferred to the series manufactured elements. Up to 2013, 409 elements of the 890 required elements have been delivered and approximately $10 \%$ have been HHF tested, indicating no problems with the bonding at $10 \mathrm{MW} / \mathrm{m}^{2}$.

These HHF tests in GLADIS used surface temperature measurement to characterize the elements, with a well-defined heat source and an optimally positioned and calibrated infrared camera. However, in W7-X surface coatings will affect the perceived surface temperature [9], the limited spatial resolution and small solid angle of the inspection cameras will make the temperature measurement less accurate and the power and power density to the elements will be difficult to accurately quantify. Given these difficulties, it is important to know that the elements can withstand short term thermal overloading on the divertor elements and structures.

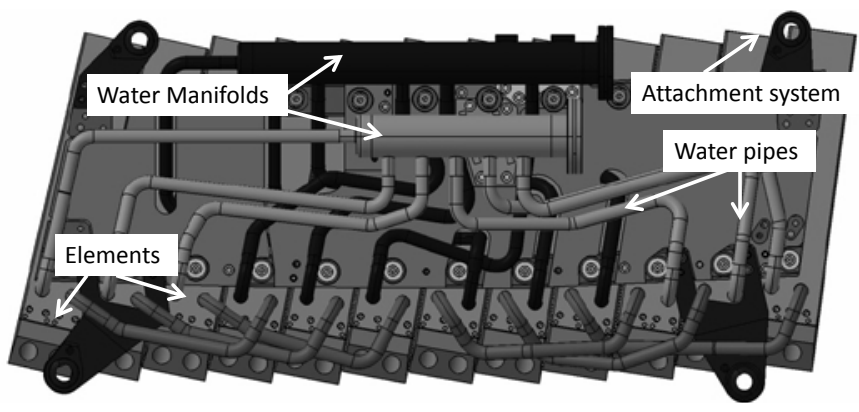

Fig. 4. View of Target Module 8 from the rear showing the cooling pipes and manifolds..

To investigate this, prototype elements have been tested at $14 \mathrm{MW} / \mathrm{m}^{2}$ and at $16 \mathrm{MW} / \mathrm{m}^{2}$ for 1000 cycles where a slow debonding of the tiles took place. The higher the load, the higher was the rate of de-bonding. However, elements damaged in this way were subsequently tested at $10 \mathrm{MW} / \mathrm{m}^{2}$ and shown to be still useable at their nominal load [10]. The elements, therefore, while sensitive to overloads have shown that they can be temporarily overloaded without significant damage. Importantly the elements show a gradual degradation of their performance under these conditions giving the chance to perform corrective measures. These tests showed that a fast de-bonding of the tiles is not expected. This outcome is also very important considering the limited number of spare elements and the difficulties that will be encountered to replace a target module or target element once these components have been installed in the plasma vessel.

One of the strategies that has been developed over the last few years has been the development of the "test divertor unit" (TDU) [11], an intermediate adiabatically cooled divertor with the same geometry as the subsequent HHF divertor. This divertor will provide a test bed for the establishment of the plasma operational scenarios and the control and diagnostic systems that will be later used for with the HHF divertor components.

Much of the development of the divertor over the last years has concentrated on the actively water-cooled HHF elements. Recently the design and development work on the modules, their water circuits and associated integration within the plasma vessel has been resumed. In order to bring the design in phase with the element production the design and development of the modules for the high iota tail of the divertor was at first undertaken.

The design of the modules has been based on the outline cooling system design and the element design defined in 2002. The detailed design of the divertor modules resumed with TMh9 followed by TMh8 and TMh7. A model of the TMh8 from the rear side showing the main features of the design: solid butt welded piping, fully welded manifold to distribute the water equally between the elements with flanged connections to the water supply system and two rails joined by a stiffening plate forming a frame onto which the elements are mounted is shown in Fig. 4. The main design challenge was to fit modules in the limited space available between the nominal target surface and the plasma vessel and other IVCs. Due to the strong 3D nature of the plasma vessel in this area it was necessary to have a complicated piping arrangement, which has the advantage that the pipes are sufficiently flexible that they can compensate for the movement of the elements under thermal load. The presence of diagnostics and cooling pipes from other components in this area also has an impact on the design of the components. The developed design achieved a gap of at least $10 \mathrm{~mm}$ to all of the neighbouring components. This is necessary to allow for the adjustment of the target to their optimal position once the machine has been assembled and the position of the magnetic field is known.

In parallel to the design activities a prototype of the module TMh9 was built using prototype target elements. This prototype module, with 12 elements, can be seen in Fig. 5 mounted in the GLADIS chamber ready for HHF testing.

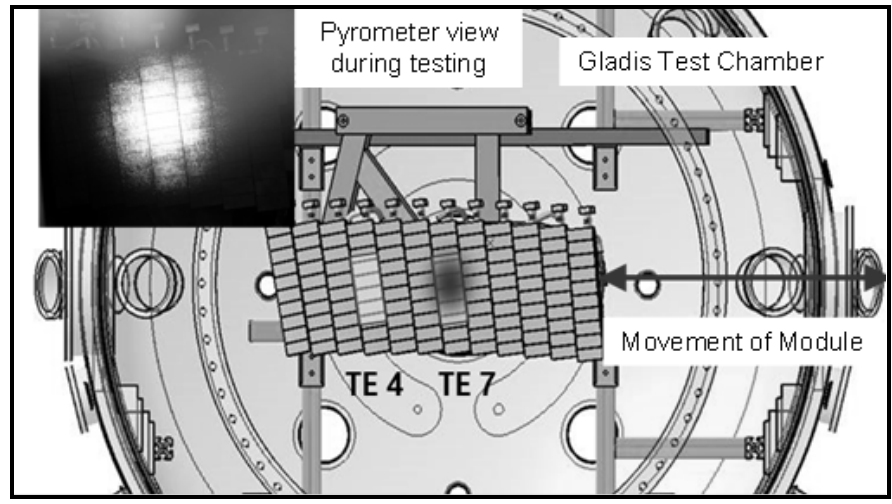

Fig. 5. Prototype module in GLADIS chamber

All of the technologies to be used in the manufacture of the modules were proved with the production and testing of the prototype. A number of improvements were identified in the manufacturing sequence which will make the series manufacturing quicker and more reliable. 
After manufacture the prototype was leak and water flow tested, meeting the required specified values. The prototype was then tested in GLADIS with fluxes up to $13 \mathrm{MW} / \mathrm{m}^{2}$ to prove that the attachment concept and the flexibility of the piping arrangement within the module could withstand the complex thermal stresses and strains during HHF loading. The prototype module was also analysed in detail using FEM calculations [12]. These calculations complemented the GLADIS tests in analysing heat load patterns which corresponded to those expected in W7-X and looked at stress distributions occurring in the components of the module. Results of the tests and the calculations were fed back into the design to improve the modules.

To form a smooth divertor surface, the top of the target elements is 3D-machined before mounting them onto the mechanical support structure of the module. One of the important features of the GLADIS test was that it tested these 3D machined target element surfaces. There appeared to be no significant effect on the temperature measurement of the target surfaces and this also demonstrated that the machining process developed left no dust or debris on the surface of the elements and there was no need for subsequent cleaning of the elements.

Following the initial detailed FEM analysis of the prototype module the critical areas were identified and the calculations repeated for the finally designed TMh7-TMh9; concentrating on those critical areas. No problems were identified and together with the results of the GLADIS tests this formed the basis of the release for manufacture of the target modules, TM7h, TM8h and TM9h, see Fig 1. The manufacture of the 3D machined elements, manifolds and modules frames has already been partially completed (June 2013). It is planned that $10 \%$ of the serial modules will be tested under HHF conditions in GLADIS to further confirm their thermohydraulic behaviour, in combination with a detailed series of tests that the modules will undergo to confirm that they correspond to their specification.

In parallel to the manufacturing of the target module TM7h, TM8h and TM9h the design of the modules in the vertical target area is continuing using the same design principles established for the already designed and qualified modules. The main design challenge here is also fitting the components into the available space envelope, which is highly constrained due to the proximity of the plasma vessel in this region and the already designed and completed components surrounding the divertor.

\section{B. High Heat Flux Divertor, Element Ends.}

The divertor target surfaces developed with the 9 "reference" plasma configurations enable W7-X to operate with a wide variety of plasmas consistent with the aims and goals of W7-X [4]. Estimates of the poloidal footprint of the strike zone of a W7-X plasma indicate that the half width of the footprint is between $40-50 \mathrm{~mm}$, as shown in Fig. 3. The elements in the main horizontal target area are typically 600 $\mathrm{mm}$ long.

This allows a significant operating space for the strike points. However, the geometry of the HHF elements does not allow the full $10 \mathrm{MW} / \mathrm{m}^{2}$ to be incident along the whole element length of the element. For approximately 25-50 mm, at both ends, the element cannot be fully loaded due to the geometry of the water connection or the internal water channel geometry of the element itself. For the last top tile on the element a limit of $5 \mathrm{MW} / \mathrm{m}^{2}$ was specified.

In the original conceptual development of the W7-X divertor this was not such a large issue since the plasma was thought to be relatively static. Interesting plasma scenarios also have the strike point as close as possible to the end of the element to maximise pumping efficiency. This is only possible with limited movement of the strike point across the target area.

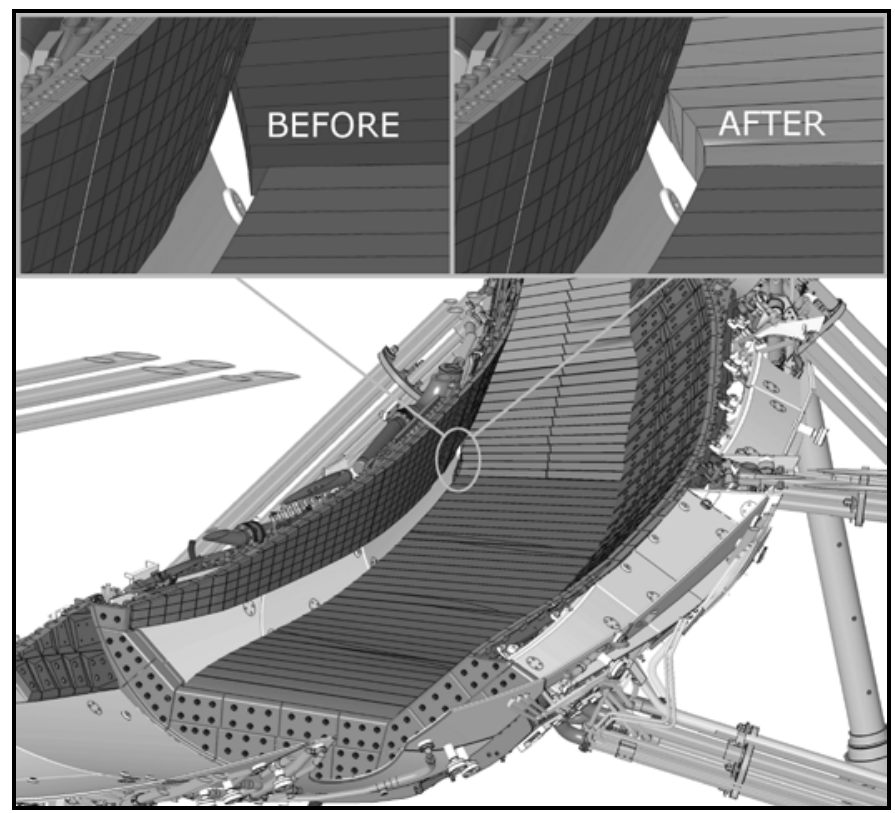

Fig. 6. Adaption of the module design at the transition TM4h/ TM5h

However, the development of computing capacity and increased understanding of how plasmas develop has led to additional configurations which possibly will challenge the presently designed components. Recent studies have postulated that bootstrap currents [13] develop resulting in a changing configuration and the movement of the strike point across the element ends. The divertor did not take such configurations into account in its design. Hence a number of actions have been taken to mitigate the effect of this type of scenario on the divertor. Modifications to the divertor itself are limited because of the advanced state of the element design and procurement. Some of the elements have been moved radially into the pumping gap to reduce the loads on the ends of the elements. In addition the ends transition between module TMh4 and TMh5 has been re-designed to avoid steps and reduce the risk of conducted power going behind the modules, see Fig. 6. Other measures, such as an additional protection component, to be built into the machine are described in the paper by Lumsdaine [14].

Experimental studies have been undertaken to see what the margin of performance of these end tiles is in comparison to the postulated loads that could be present, either as a result of standard configurations or of the new bootstrap current defined 
variants. These tests in GLADIS are shown in Fig 7. Tests have been performed, without tile detachment but with some bonding degradation, up to $7.5 \mathrm{MW} / \mathrm{m}^{2}$ for 10 pulses for the top end tile and $4 \mathrm{MW} / \mathrm{m}^{2}$ for the end tile compared with 5 $\mathrm{MW} / \mathrm{m}^{2}$ and $2 \mathrm{MW} / \mathrm{m}^{2}$ specified, respectively. The margins are comparable to those of the normal top tiles, i.e. not enough to match the new requirements, in excess of $10 \mathrm{MW} / \mathrm{m}^{2}$.

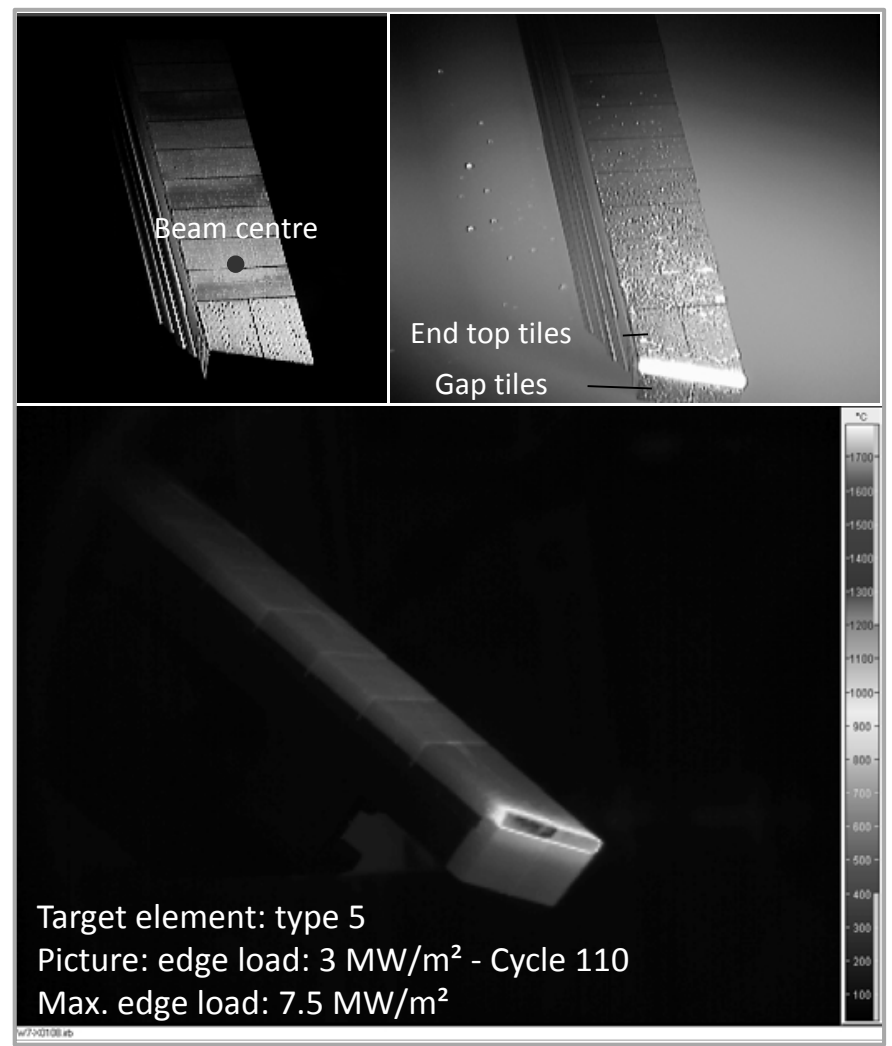

Fig. 7. Element loaded in GLADIS at $45^{\circ}$ showing strong end illumination.

Fig. 7 shows the element angled at $45^{\circ}$ to the beam to test both the end top tile and the end tile simultaneously. The elements are tested in the state that they are delivered by the manufacturer Plansee, with CFC of uniform thickness along and across the element. In order to match the 3D contours of the divertor surface each element will be 3D machined: the top surface as well as the end facing the pumping gap. This reduces the thickness of the CFC, and the total length of the element slightly. The reduction in length of the element means that it should be able to withstand higher fluxes; however, this must be experimentally confirmed. The design of this end tile could also allow its overloading to be easily observed by the in-vessel video diagnostics.

Near to the water connectors the geometry of the element changes and the distance from the tiles to the cooling water increases. This can lead to a higher interface temperature for the same nominal loads and a reduction in their performance. The testing in GLADIS showed that these tiles would have a limit of approx. $8 \mathrm{MW} / \mathrm{m}^{2}$. The limitation of the water connection end of the element has been taken account into the design of the horizontal targets, as it is covered with the baffle as shown in Fig. 8. However, for the vertical targets they are directly exposed to the plasma and operational means have to be found to keep the strike points away from this region. The strike points from the reference plasmas do not come near this region.

During series testing in GLADIS the two tiles adjacent to the water connectors are tested only with $8 \mathrm{MW} / \mathrm{m}^{2}$.

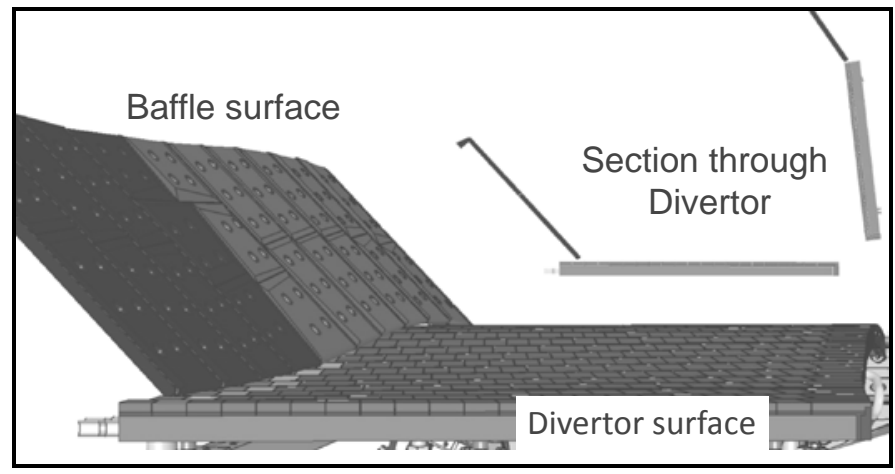

Fig. 8. Protection of the elements end by the baffle

\section{Low Heat Flux Divertor}

The divertor target modules, TM5h and TMh6, have a total area of $6 \mathrm{~m}^{2}$ and were originally conceived to be high heat flux modules. This was changed to be a variant with a performance between the divertor and the baffle. The reduction in performance of this area was realized by recessing it below the other target modules and re-defining the surfaces of the remaining HHF modules. The present design of these modules is shown in Fig. 9.

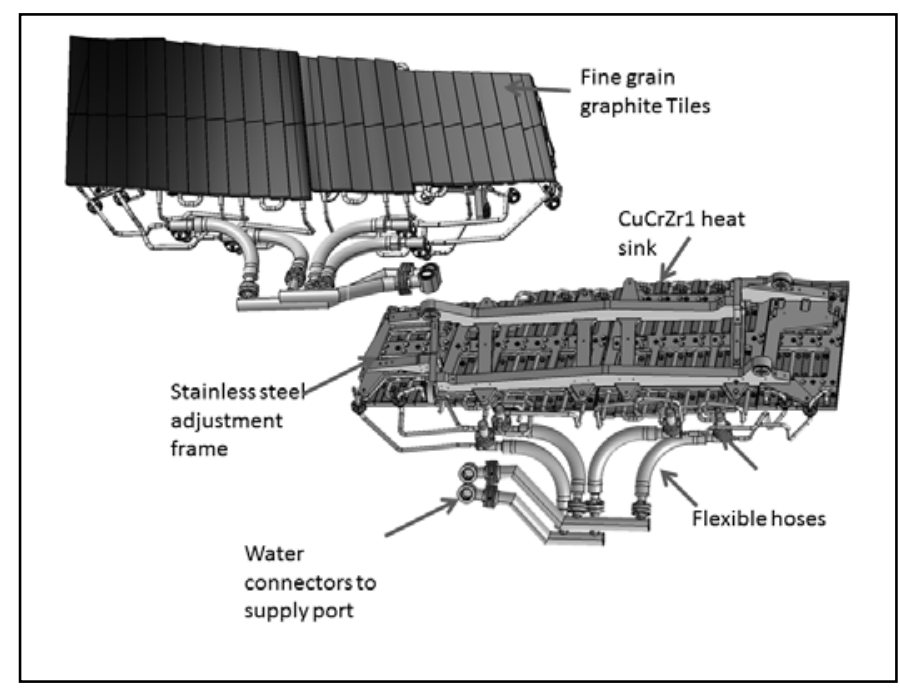

Fig. 9. Present design of low heat flux divertor (TM5h and TM6h).

The design uses heat shield technology. The tiles are attached from the rear to avoid holes in the plasma facing surface. Another feature of the design is the overlapping heat sinks, which avoids excessive radiation loads from the hot tiles entering the gap between the heat sinks and overheating the attachment structure and the vacuum vessel. The specified load for this component is $1 \mathrm{MW} / \mathrm{m}^{2}$ over a poloidal extent of 100 
$\mathrm{mm}$, this is more than the expected poloidal extent of the footprint defined in section II.B for the horizontal target.

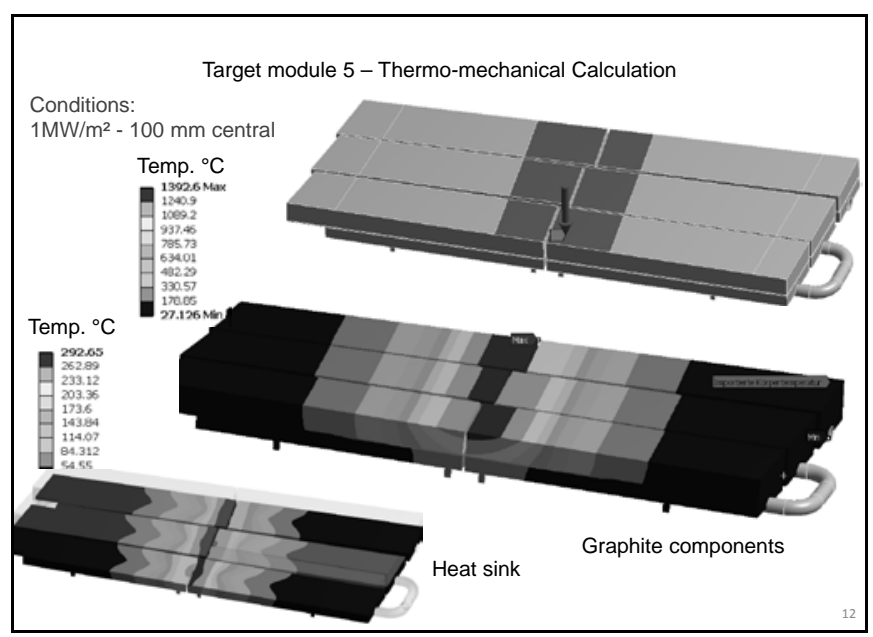

Fig. 10. FEM calcualtion of the LHF component: temperature distribution.

The heat loads are achieved by using thicker graphite tiles than used for the baffle. The thicker graphite also gives additional protection for thermal overloads over short periods of time but has the disadvantage that significantly higher graphite temperatures are expected than for the baffle.

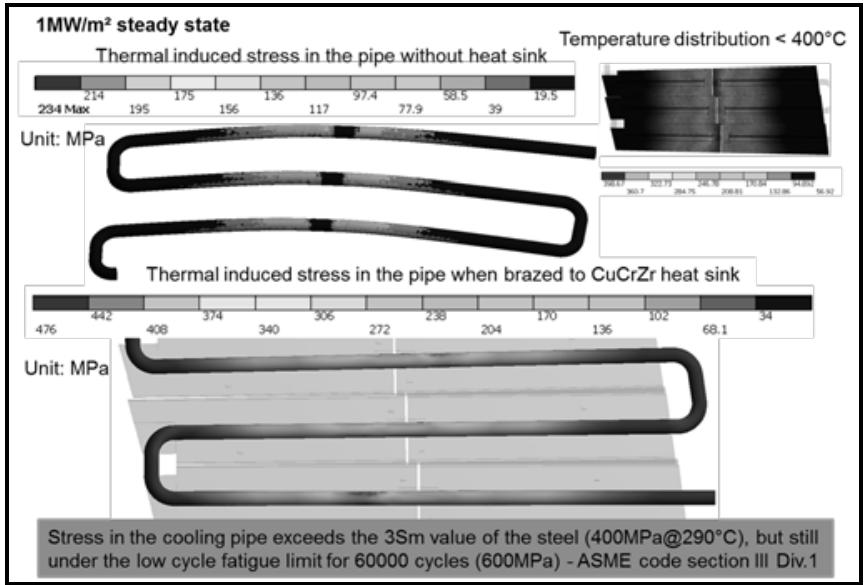

Fig. 11. Stress analysis in the pipe-work of the heat sinks. The pipe inner diameter is $10 \mathrm{~mm}$ and its thickness $1 \mathrm{~mm}$.

FEM analysis of these components has been performed and confirmed the desired performance limits. Fig. 10 shows the temperature evolution of the surface and across the joint between the CuCrZr heat sink and the cooling tube for the specified operational loading. This region was chosen for the analysis because it covers the transition between the two heat sinks and is expected to be the location which gives the highest stresses. Fig.11 shows the resulting thermo-mechanical analysis. The analysis indicates that the highest stresses occur at the connection of the stainless steel pipe to the CuCrZr heat sink.

These thermal stresses are a general feature of this type of technology and have been minimized by optimizing the positioning of the fixed points and sliding supports of the heat sinks. In this design there is little margin compared to the specified value but the heat load footprint is conservative. Analysis with $6 \mathrm{MW} / \mathrm{m} 2$ indicates that either a narrower footprint, shorter time or a limitation to the number of cycles at this load will be necessary.

In addition to the thermo-mechanical analysis, flow analysis has also been performed. This uses the Flowmaster ${ }^{\mathrm{TM}}$ code [15] to calculate the flow in the different branches of the parallel cooling system to ensure that an overall minimum flow velocity of $6 \mathrm{~m} / \mathrm{s}$ is maintained and to ensure that the variation in flow between the different branches is not too high. This is necessary to minimize the total water flow requirement and to ensure that the water flow velocity does not become too high in the branches where the highest flow exists and in the flexible hoses which are used to connect the components to the plugins. These flexible hoses are necessary to allow for thermal expansion and for installation tolerances. The geometry of the cooling tubes is shown in Fig. 12.

Balancing is achieved by designing the component branches to have equal hydraulic lengths, in so far as the geometrical constraints allow, performing the analysis, seeing if the resulting flow is sufficiently uniform and then if not orifices will be introduced at the design stage to redistribute the flow. This analysis is performed based on the nominal CAD geometry of the components and pipework. These analysis results will be later compared with actual measurements of the components once they have been manufactured. Normally for components such as these with smooth bends and relatively simple geometries there is very good correlation between measurement and analysis [16]. However, if the water flow measurements do show an unacceptable variation in the flow velocity then further orifices will be introduced into some of the cooling branches to finally balance the flow. The advantage of the Flowmaster ${ }^{\mathrm{TM}}$ software is that it allows measured component flow properties to be included in the network and defines the properties of the orifices needed. Space has been left within the module design for the inclusion of these orifices if they are needed.

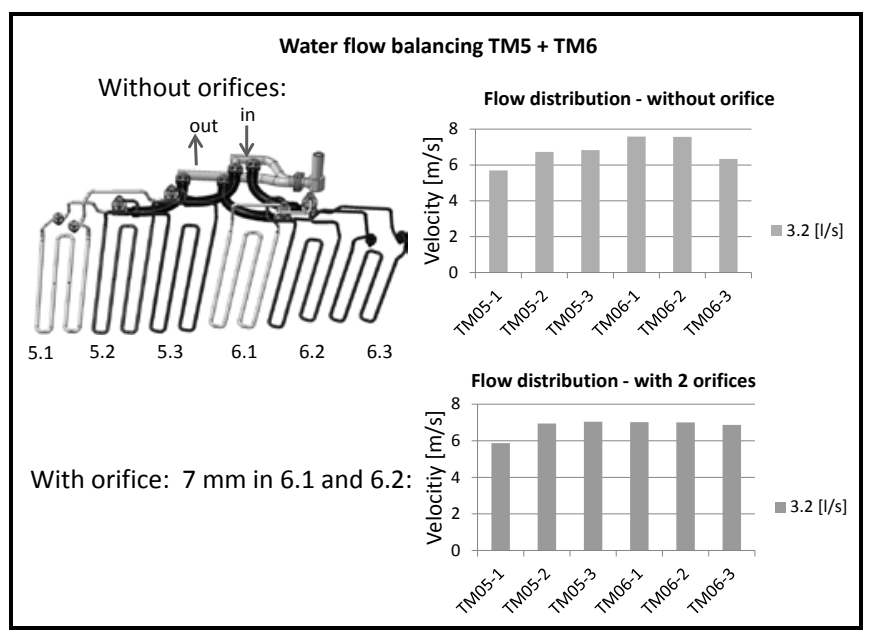

Fig. 12. Flow distribution with and without orifices. TMh5-1, 2,,3 and TMh61-2-3 are the three meanders placed in parallel in each module. 
The balancing of hydraulic circuits with components of very different geometries has been found to be one of the most difficult problems to be addressed especially in the W7-X geometry, which because of the limited number of feedthroughs and the large surface area to be cooled needs to have highly parallel coolant flow.

The pipework for the TM5h and TM6h modules, apart from the flexible hoses, uses fixed pipework. This pipework has been checked to ensure it has enough flexibility to allow for thermal movements. This has been demonstrated by combined thermal-mechanical FE analysis. Stresses of the order of 60 $\mathrm{MPa}$ have been found in the pipes for conservative load assumptions.

The completion of every component design is concluded by a complete configuration check [17] to ensure that there are no unforeseen collisions with other components.

\section{SHINE THROUGH AREAS}

There are two neutral beam systems in the W7-X machine. The first and most powerful, the NBI system, is used for plasma heating. Details of the heating NBI system can be found in [18]. The second system is the so-called diagnostic NBI system (DNBI) which is described in [19]. Both of these systems inject significant amounts of power into the vessel and in principle the vast majority of this power should be absorbed by the plasma. However, if for a number of reasons the plasma is not present or the plasma has a low density, then either the full heating load or part of it will fall onto the IVCs. Assessments have been performed and countermeasures have been taken to mitigate the possible consequence of the shinethrough of both systems. The maximum loads to the IVCs are summarised in Table I. All of the shine through areas and graphite port protections will be water cooled when the beams are in operation.

TABLE I. EFFECT OF NBI SYSTEMS ON THE IVC.

\begin{tabular}{|c|c|c|c|c|}
\hline System & $\begin{array}{l}\text { Total } \\
\text { Power } \\
\text { MW }\end{array}$ & $\begin{array}{l}\text { Peak heat } \\
\text { load to IVC } \\
\mathrm{MW} / \mathrm{m}^{2}\end{array}$ & $\begin{array}{l}\text { Components } \\
\text { Affected }\end{array}$ & $\begin{array}{c}\text { Construction } \\
\text { Graphite/ } \\
\text { CuCrZr }\end{array}$ \\
\hline DNBI & 0.3 & 13 & 1 & Graphite tiles \\
\hline $\mathrm{NBI}$ & 20 & 43 & 6 & Graphite tiles \\
\hline
\end{tabular}

\section{A. Neutral Beam Heating System}

The NBI system has the capability of injecting up to 20 MW of NBI power into W7-X through two axisymmetric ports, each with four NBI sources. The expected footprint of the four beams on the opposite wall is shown in Fig. 13. This simulation assumes that there is an empty machine and the effects of beam re-ionisation and beam scattering have not been taken into account. The presence of a plasma will significantly reduce the shine-through. As can be seen from Fig. 13 the beam shine-through hits areas of the baffles, heat shields, toroidal closure plates and the divertor surface.

The peak unprotected load is $43 \mathrm{MW} / \mathrm{m}^{2}$ in the centre of one of the four main beam profiles. The exact value varies slightly as the angle of incidence is slightly different for each source and the transmission from each source through the ports is slightly different. Loads of this magnitude are planned on the IVC for fractions of a second to calibrate the interlocks.

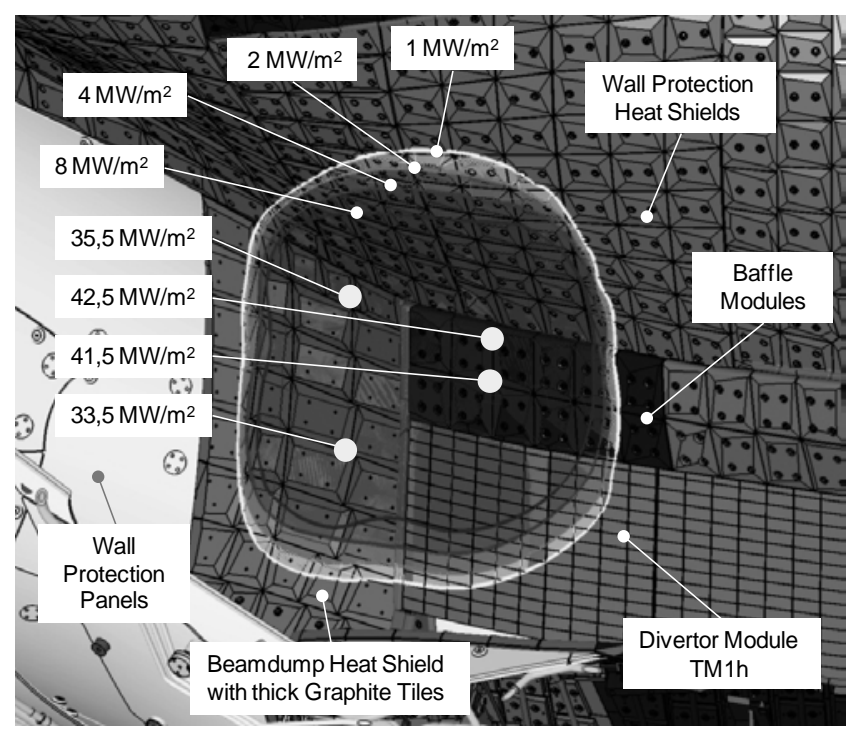

Fig. 13. Contours of $1 / 2 / 4 / 8 \mathrm{MW} / \mathrm{m}^{2} \mathrm{NBI}$ beam power on IVCs (on axis view).

Graphite and CFC were both assessed to be used in this area. Graphite was selected after the thermal stresses were assessed to be within acceptable limits.

In order to maximize the short period loads that can be accepted by these components the graphite has been made thicker, $20 \mathrm{~mm}$ instead of $10 \mathrm{~mm}$ and the tile attachment system has been modified to allow front access without significantly exposing the TZM bolts used to attach the tiles to the cooling structure to the high energy beam particles.

A detailed assessment of the shine-through fraction as a function of plasma density has been performed. In the first operation phases of the W7-X machine only hydrogen (protium) will be used as the beam species and the assessment is based on this beam. The use of deuterium at later stages of operation will allow higher beam power but, since the absorption of deuterium beams in the plasma is higher, then the overall effect on the shine-through region is similar. Fig. 14 shows a study of the potential limit to the NBI pulse length by postulating certain thermal limits in the IVC of the shinethrough region.

The graph summaries calculations of the shine-though fraction for different densities and then for this shine through fraction assesses how long the pulse can last before limits are experienced in the IVCs. For the longer pulse lengths this limit may be reached after the end of the NBI pulse since the energy stored in the tile may cause the heat sink to overheat after the pulse. Nominally the beam should be able to operate for 10 seconds but this may be limited at lower plasma densities by the effect of the shine through fraction on the IVC.

For very low plasma density this limit is due to the graphite surface temperature. For intermediate plasma densities the 
limit changes to the total heat input into the IVC causing overheating of the copper heat sink. Based on the technology used for these components, there is little that can be done to improve the situation. CFCs with higher conductivity improve the situation at lower densities but increase the heat transfer to the heat sinks causing their overheating at longer pulse time. Other changes have been proposed for the components in this area but these have a negative effect on the performance of the components under long pulse operation.

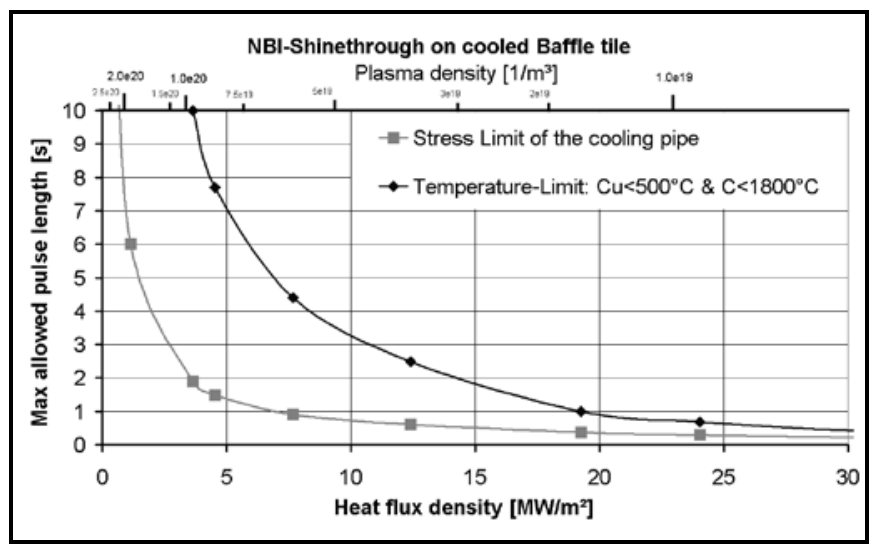

Fig. 14. Maximum allowed pulse length based on different limits.

The assessments here are based on a number of assumptions. Using these the IVCs could limit the allowed beam time at low plasma densities. Instrumentation of this area has been implemented to measure the effects of the shine through. Additionally operation of the machine will indicate if there is design freedom to, for instance, increase the thickness of the graphite tiles. This will increase the inertial component of the graphite tile and allow cooling between beam shots.

\section{B. Diagnostic Neutral Beam Heating System}

The DNBI system, used to characterise the W7-X plasma, operates similarly to the NBI system apart from having a much lower performance (300 kW for $5 \mathrm{~s}$ ) and only one source. However, the parameters of the beam are such that a significant energy density falls on the IVCs. The expected heat load to the IVCs is $13 \mathrm{MW} / \mathrm{m}^{2}$ at a plasma density of $3.10^{19} \mathrm{~m}^{-3}$. Here similarly to the NBI system the graphite shine through tiles have an increased thickness and are instrumented to check they do not over-heat.

\section{CONCLUSIONS}

The HHF divertor components of W7-X continue to be designed, developed and produced. Geometric considerations continue to dominate the design requirements and technological progress has been made in a number areas.

Assessments and experiments have shown that the HHF divertor components have some margin over their specified loading capability. Simulations of different plasma scenarios and heating conditions have also allowed a better identification of the location and heat load specification of IVCs.

W7-X now needs to go into operation to define which plasma scenarios are of interest, which heating schemes work and to define what the real loads to the plasma facing components are. The IVCs have been designed according to the specified load conditions based on a set of reference plasma scenarios and in particular are compatible with steady state divertor operation of $10 \mathrm{MW} / \mathrm{m}^{2}$.

Using the portfolio of available standard designs and technologies used for W7-X, design modifications have improved the performances of the components at critical locations while trying to minimize restrictions on operation. When required, operation limits have been identified; such as for the NBI system.

\section{REFERENCES}

[1] R. König, J. Baldzuhn, C. Biedermann, R. Burhenn, S. Bozhenkov et al., "Diagnostic development for quasi-steady-state operation of the Wendelstein 7-X stellarator”, Rev. Sci. Instrum. 83, 10D730 (2012).

[2] R. Stadler, A. Vorköper, J. Boscary, A. Cardella, F. Hurd, C. Li, et al., "The in-vessel components of the experiment Wendelstein 7-X", Fusion Engineering and Design, vol. 84, no. 2-6, pp. 305-308, June 2009.

[3] H. Greuner et al, "Structure of divertor for the optimised stellarator W7X”. Fusion Technology, pp. 323-326, 1994.

[4] J. Kisslinger et al, "Divertor Concept for the WENDELSTEIN 7-X Stellarator: Theoretical Studies of the Boundary and Engineering”, EPS Lisboa (1993), 17C, II, 587.

[5] H. Renner, J. Boscary et al., "The capabilities of steady state operation at the stellarator W7-X with emphasis on divertor design”, vol. 40, no. 6, Nucl. Fus., pp. 1083-1093, June 2000.

[6] M. Lipa, J. Schlosser, and F. Escourbiac, "Development of Actively Cooled Plasma Facing Components for Tore Supra”, Fus. Sci. Techn., vol. 56, no. 3, pp. 1124-1149, October 2009.

[7] H. Greuner et al, "High heat flux facility GLADIS: Operational characteristics and results of W7-X pre-series target tests”, J. Nucl. Mater. 367-370 1444-8, 2007.

[8] H. Greuner, U. v. Toussaint, B. Böswirth, J. Boscary, H. Maier, A. Peacock et al, "Performance and statistical quality assessment of CFC tile bonding on the pre-series elements of the Wendelstein 7-X divertor", Fus. Eng. Des., vol. 86, no. 9-11, pp. 1685-1688, October 2011.

[9] D. Hildebrandt, A. Dübner, H. Greuner and A. Wiltner, "Thermal response to heat fluxes of the W7-AS divertor surface submitted to surface modification under high temperature treatment”, Journal of Nuclear Materials 390-391 (2009) 1118-1122.

[10] H. Greuner, B. Böswirth, J. Boscary, T. Friedrich et al., "Review of the high heat flux testing as an integrated part of W7-X divertor development” .Fus. Eng. Des., vol. 84, no. 2-6, pp.848-852, June 2009.

[11] A. Peacock, H Greuner, F.Hurd, R König, B Mendelevitch, R Stadler, et al, "Progress in the design and development of a test divertor (TDU) for the start of W7-X operation”, Fusion Eng. Design, vol. 84, no. 7-11, pp.1475-1478, June 2009.

[12] X. B. Peng et al., "Thermo-mechanical analysis of Wendelstein 7-X plasma facing components", "in press".

[13] J. Geiger et al., "Physics Modeling for Steady-state Experiments at Wendelstein 7-X”, 23rd IAEA Fusion Energy Conf. 2010, THC-P2-02.

[14] A. Lumsdaine, et al., "Wendelstein 7-X high heat-flux divertor scraper element", this conference.

[15] www.flowmaster.com

[16] M. Smirnow, G.Orozco, J. Boscary and A. Peacock, "Hydraulic Analysis of the Wendelstein 7-X cooling loops”, "in press”.

[17] J. Tretter, J. Boscary, B. Mendelevitch, A. Peacock, R. Stadler "Configuration Space Control of In-Vessel Components for Wendelstein 7-X", this conference

[18] McNeely, P.; Barlak, M.; Baldzuhn, J.; Bozhenkov, S.; Gawlik, G.; Heinemann, et.al., Current Status of the Neutral Beam Heating System of W7-X, 27th Symp. on Fusion Technology (SOFT ), 2012, Liège.

[19] T. Richert et al. "Diagnostic neutral beam injector for Wendelstein 7X", SOFE Conf. 2013 San Francisco, to be published. 
